\title{
ПРОСТРАНСТВЕННО-ВРЕМЕННАЯ ГОЛОГРАФИЧЕСКАЯ ИНТЕРФЕРОМЕТРИЯ
}

A. REBANE, J. AAVIKSOO. AJALIS-RUUMILINE HOLOGRAAFILINE INTERFEROMEETRIA

A. REBANE and I. AAVIKSOO. TIME-SPACE HOLOGRAPHIC INTERFEROMETRY

\section{(Представил К. К. Ребане)}

Рассматривается способ интерферометрического исследования меняющихся сверхбыстро во времени световых сигналов на основе развитого в последние годы в Институте физики АН ЭССР метода пространственно-временной голографии в средах с фотовыжиганием спектральных провалов (ФСП).

По аналогии с традиционной пространственной голографической интерферометрией $\left[{ }^{1}\right]$, суть предлагаемого способа заключается в когерентном сравнении амплитуд двух записанных посредством голограммы световых сигналов с целью выявления существующего между ними малого различия.

Однако в рассматриваемом в данной работе случае в отличие от традиционного подхода, где посредством интерферограмм воспроизводятся и сравниваются лишь пространственные характеристики световых амплитуд, в предлагаемом способе интерферометрический анализ распространяется за счет голографической записи временных амплитуд и на временные и спектральные характеристики световых сигналов. Основой для этого служит продемонстрированная в $[2,3]$ способность ФСПголограмм записывать и воспроизводить одновременно как пространственные, так и временные зависимости амплитуды рекордно коротких световых сигналов длительностью вплоть до 100 фсек [ $\left.{ }^{4}\right]$.

В данной работе рассматривается т. н. двухэкспозиционная схема голографической интерферометрии. Предполагается, что на одной и той же пластинке-голограмме, изготовленной из соответствующей среды с фотовыжиганием спектральных провалов, осуществляется запись двух близких друг к другу пространственно-временных голографических образов меняющейся быстро во времени сцены или сигнала. Далее показано, что, применяя для считывания голограммы перестраиваемый по частоте узкополосный лазер, можно эффектвно выявить малую разницу между сравниваемыми пространственно-временными сценами.

Пусть тонкая пластинка-голограмма из регистрирующей ФСП-среды, расположенная в плоскости $(x, y)$, облучается в момент времени $t=0$ со стороны $z>0$ коротким референтным импульсом с $\delta$-образным амплитудным временным профилем и с плоским волновым фронтом, нормаль которого образует с нормалью плоскости голограммы угол $\theta$. Представим амплитуду референтного импульса в плоскости пластинки-голограммы в виде

$$
R(\vec{r}, t)=R_{0} \delta(t+x \theta / c) \exp \left[i \omega_{0}(t+x \theta / c)\right],
$$

где $\omega_{0}-$ несущая оптическая частота и $c-$ скорость света.

Предположим, что вслед за референтным импульсом пластинка-голограмма облучается также со стороны $z>0$ сигнальным импульсом длительностью $t_{s}$, пространственно-временная амплитуда которого суть исследуемая сцена или событие. Амплитуда сигнального импульса в плоскости $(x, y)$ меет вид

$$
S(\vec{r}, t)=s\left(x, y, t-t_{R}\right) \exp \left[i \omega_{0}\left(t-t_{R}\right)\right],
$$


где $t_{R}$ - временная задержка сигнального импульса, относительно референтного импульса.

Как и ранее $[2,3]$, будем считать, что оптическая частота рабочей неоднородно-уширенной полосы поглощения регистрирующей ФСПсреды совпадает с оптической частотой сигнала $\omega_{0}$ и что спектральная ширина неоднородной полосы намного больше спектральной ширины сигнального импульса. Будем считать также, что однородная спектральная ширина $v_{\text {н }}$ чисто электронной линии ФСП-молекул регистрирующей среды достаточно мала, так что выполняется условие

$$
\Delta v_{\mathrm{H}} \ll t_{R}^{-1}<t_{\mathrm{S}}^{-1} \text {. }
$$

Совместное воздействие референтного и сигнального импульсов на регистрирующую ФСП-среду приводит к записи пространственно-временной голограммы, амплитудный пространственно-спектральный коэффициент пропускания которой можно в линейном приближении $\left[{ }^{2,3}\right]$ выразить как

$$
\begin{gathered}
K^{(1)}(x, y, \omega) \sim(1+i \widehat{H})\{I(x, y, \omega)\}= \\
(1+i \widehat{H})\left\{R_{0}^{2}+\left|\bar{s}\left(x, y, \omega-\omega_{0}\right)\right|^{2}+R_{0} \bar{s}\left(x, y, \omega-\omega_{0}\right) \exp \left[-i \omega\left(x \theta / c+t_{R}\right)\right]+\right. \\
\left.+R_{0} \bar{s}^{*}\left(x, y, \omega-\omega_{0}\right) \exp \left[i \omega\left(x \omega / c+t_{R}\right)\right]\right\},
\end{gathered}
$$

где $\bar{s}$ обозначает фурье-образ амплитуды (2), а наличие преобразования Гильберта

$$
\widehat{H}=\{f(\omega)\} \equiv \frac{1}{\pi} \int f\left(\omega^{\prime}\right) d \omega^{\prime} /\left(\omega^{\prime}-\omega\right)
$$

гарантирует удовлетворение со стороны комплексной функции отклика $K^{(1)}(x, y, \omega)$ принципа причинности.

Используем далее эту же пластинку для записи второй пространственно-временной голограммы этой же сцены или сигнала, несколько отличающегося, однако, от предыдущего образа как по пространственной, так и по временной структуре. Выразим амплитуду второго сигнального импульса в плоскости голограммы в виде

$$
\bar{s}^{\prime}\left(x, y, \omega-\omega_{0}\right)=\bar{s}\left(x, y, \omega-\omega_{0}\right) \exp [i \varphi(x, y, \omega)],
$$

где функция $\varphi(x, y, \omega)$ заключает в себе различие между двумя рассматриваемыми сценами. Полезно также предполагать, что при второй экспозиции угол наклона референтного импульса $\theta^{\prime}$ и его временное опережение $t_{R}$ по отношению к сигнальному импульсу несколько отличаются от значений, соответствующих первой экспозиции, так что $\Theta^{\prime}=\Theta+\Delta \Theta$ при $\Delta \Theta \ll \Theta$ и $t_{R}^{\prime}=t_{R}+\tau$.

B итоге записи двух экспозиций амплитудное пространственноспектральное пропускание пластинки-голограммы без учета несущественных с точки зрения данной задачи членов выражается как

$$
\begin{aligned}
& {\left[K^{(1)}(x, y, \omega)+K^{(2)}(x, y, \omega)\right] \sim } \\
\sim & R_{0} \bar{s}\left(x, y, \omega-\omega_{0}\right) \exp \left[-i \omega\left(x \theta / c+t_{R}\right)\right]+ \\
+ & R_{0} \bar{s}^{\prime}\left(x, y, \omega-\omega_{0}\right) \exp \left[-i \omega\left(x \theta^{\prime} / c+t_{R}^{\prime}\right)\right],
\end{aligned}
$$

т. е. содержит полную пространственно-временную информацию о двух коррелированных между собой событиях.

Для анализа интерферограммы предположим, что пластинка с записью двух голограмм облучается плоскопараллельным пучком узкополосного непрерывного лазера с оптической частотой $\Omega$ под углом $\Theta^{\prime \prime} \approx \Theta^{\prime}, \Theta$. Считывающий пучок одночастотного лазера испытывает дифракцию одновременно на обеих записанных голограммах и воспроизводит два интерферирующих друг с другом пространственных мнимых изображения. Непосредственно за голограммой интенсивность дифрагированного пучка выражается как

$$
\begin{gathered}
I(x, y, \Omega) \sim I_{0}(\Omega) R_{0}^{2}\left|\bar{s}\left(x, y, \Omega-\omega_{0}\right)\right|^{2} \times \\
\times 2\{1+\cos [\varphi(x, y, \Omega)-\alpha(x, y, \Omega)]\}
\end{gathered}
$$


где $I_{0}(\Omega)$ - интенсивность считывающего пучка и

$$
\alpha(x, y, \Omega) \equiv \Omega x \Delta \theta / c+\Omega \tau .
$$

Как и в случае традиционной голографической интерферометрии, в пространственном распределении интенсивности дифрагированного пучка (8) обнаруживается набор интерференциониых полос. Период этих полос в пространстве пропорционален $\Delta \Theta^{-1}$. Пространственные неоднородности данных интерференционных полос соответствуют пространственно-неоднородным изменениям оптической длины пути для лучей сигнальных импульсов на частоте $\Omega$ между двумя экспозициями. Подчеркнем, что в отличие от традиционного в интерферометрии подхода, при записи голограмм в средах с ФСП вообще не встает необходимости непосредственного перекрывания во времени между сигнальным и референтным импульсами.

Тем самым открывается возможность для получения четких пространственных интерферограмм даже если для их записи применяются сверхкороткие лазерные импульсы, обладающие крайне малой длиной когерентности порядка $10^{-2}-10^{-4} \mathrm{~cm}$.

Более того, благодаря сканированию частоты $\Omega$ считавыющего узкополосного лазера открывается уникальная возможность проследить и измерить нерегулярность разницы оптических фаз двух сцен для всех оптических частот сигнального светового поля.

Из (8), (9) следует, что спектральная неоднородность разности фаз выступает на фоне второго набора интерференционных полос, наблюдаемых в сей раз в спектральном диапазоне и обладающих спектральным периодом $\tau^{-1}$, т. е. определяемым разницей задержек $t_{R}$ и $t_{R}^{\prime}$.

Путем анализа искажений периодичности и контрастности спектральных интерференционных полос можно, следуя аналогии с традиционной пространственной интерферометрией, точно определить разницу во временной и спектральной структуре между двумя сравниваемыми сценами. Подчеркнем, что в данном случае спектральный (временной) анализ осуществляется в отдельности в каждой пространственной точке изображения, что создает уникальную возможность для исследования пространственно-временной структуры сигнала. В частности, можно указать, что отклонение от периодичности по частоте спектральных интерференционных полос при сканировании частоты считывающего лазера соответствует наличию нелинейной фазовой модуляции разностного сигнала, а пространственное распределение этих спектральных неоднородностей соответствует пространственному распределению характера данной нелинейной модуляции оптической фазы.

Таким образом, предлагаемый в данном сообщении интерферометрический подход позволяет, в принципе, сравнивать и с большой точностью выявлять различия между сверхкороткими широкополосными импульсными световыми сигналами пико- и субпикосекундного диапазона времени, включая амплитудную и частотную модуляцию, а также пространственные характеристики этих величин.

Авторы выражают искреннюю благодарность К. К. Ребане за предложение темы данной работы и за полезное обсуждение рукописи.

\section{Л ИТЕ Р А Т У Р А}

1. Островский Ю. Н., Бутусов М. М., Островская Г. В. Голографическая интерферометрия. М., Наука, 1980.

2. Саари П., Ребане А. // Изв. АН ЭССР. Физ. Матем., 1984, 33, № 3, 322-332.

3. Saari, P., Kaarli, R., Rebane, A. // J. Opt. Soc. Amer., 1986, B3, 527.

4. Rebane, A., Aaviksoo, J., Kuhl, J. // Appl. Phys. Lett. (в печати).
Ннститут физики
Академии наук Эстонской ССР
Поступила в редакцию $31 / \mathrm{V} 1988$ 